\title{
Avaliação de Desempenho na Comunicação em Redes Acústicas Aquáticas Utilizando Modems Reais de Baixo Custo
}

\author{
Thales T. Almeida ${ }^{1}$, Lucas Bragança ${ }^{1}$, Rafael Antônio ${ }^{2}$ \\ Francisco Lima ${ }^{2}$, Marcos A. M. Vieira ${ }^{2}$, Jose G. R. Júnior ${ }^{3}$ \\ Luiz F. M. Vieira ${ }^{2}$ e José A. M. Nacif ${ }^{1}$ \\ ${ }^{1}$ Universidade Federal de Viçosa (UFV) \\ Florestal, MG - Brasil \\ ${ }^{2}$ Universidade Federal de Minas Gerais (UFMG) \\ Belo Horizonte, MG - Brasil \\ ${ }^{3}$ Centro Federal de Educação Tecnológica de Minas Gerais (CEFET-MG) \\ Leopoldina, MG - Brasil \\ \{thales.almeida, lucas.braganca, jnacif\}@ufv.br \\ \{rafael.antonio, francisco.lima, mmvieira, lfvieira\}@dcc.ufmg.br \\ jgrjunior@leopoldina.cefetmg.br
}

\begin{abstract}
Water networks have many potential applications, such as preservation of the ecosystem, and prevention of environmental disasters. As radiofrequency signals are quickly absorbed underwater, these networks use the acoustic signal as a communication system. Compared to terrestrial networks, acoustical networks have unique characteristics, such as low bandwidth, high latency and large delay spread. With so many restrictions, one should emphasize the influence exerted by acoustic modems, whose characteristics can affect network performance. Since low-cost modems are more affordable, a good understanding of its operation is necessary. This article aims to analyze the performance of acoustic communication from the comparison between the theoretical and actual transmission rates (used in simulation experiments) and the actual transmission rates obtained for a low cost modem. Experiments were performed, where it was possible to identify the deterioration in modem transmission rates, caused by the effects of propagation delays in the medium.
\end{abstract}

Resumo. Redes aquáticas possuem diversas aplicações potenciais, como preservação do ecossistema e prevenção de desastres ambientais. Como sinais de radio-frequência são rapidamente absorvidos debaixo d'água, estas redes utilizam o sinal acústico como sistema de comunicação. Comparada com redes terrestres, redes acústicas possuem características únicas, como baixa largura de banda, alta latência e grande atraso de propagação. Com tantas restrições, deve-se ressaltar a influência exercida por modems acústicos, cujas características podem afetar o desempenho da rede. Uma vez que modems de baixo custo são mais acessíveis, se faz necessária uma boa compreensão acerca de seu funcionamento. Este artigo tem por objetivo analisar a performance da comunicação acústica a partir da comparação entre as taxas de transmissão teóricas e reais obtidas por um modem de baixo custo. Experimentos reais foram realizados, onde foi possivel identificar o comprometimento nas taxas de transmissão, deteriorada pelos efeitos dos atrasos de propagação no meio. 


\section{Introdução}

Redes aquáticas apresentam potencial para uso em várias aplicações, incluindo o monitoramento de derramamento de óleo, monitoramento de lagos e de reservatórios de hidrelétricas [Vieira et al. 2012]. Como os sinais de rádio-frequência são rapidamente absorvidos debaixo d'água, estas redes utilizam enlaces acústicos para se comunicar, uma vez que a propagação acústica é melhor suportada em baixas frequências. No entanto, apesar de apresentar um baixo custo de instalação e manutenção, a largura de banda disponível é extremamente limitada, apresentando baixíssima velocidade quando comparada com outros meios de propagação, como a propagação óptica e eletromagnética. Em transmissões acústicas, a velocidade de propagação do som alcança uma taxa máxima de 1.500 $\mathrm{m} / \mathrm{s}$. Características como a perda de transmissão, o alto nível de ruído, a propagação em múltiplos caminhos, o efeito Doppler e o atraso de propagação variável tornam a comunicação acústica ainda mais complexa [Stojanovic and Preisig 2009].

Redes acústicas aquáticas podem operar utilizando Veículos Autônomos Aquáticos (VAA) ou sensores para monitoramento, usando enlaces acústicos para comunicação. A rede pode ser ligada a uma estação central, responsável por conectá-la ao mundo externo. Esta estação pode ainda estar conectada a um backbone, que permite a conexão da rede à Internet. Este cenário possibilita que dados coletados sejam acessados remotamente, em tempo real, por cientistas e pesquisadores envolvidos em projetos de monitoramento. No entanto, a área de monitoramento destes equipamentos é limitada pelo raio de cobertura do modem acústico, que varia de poucos metros a poucos quilômetros [Catipovic et al. 1993].

Além da limitação da área de monitoramento, outro ponto relevante se refere à transmissão dos modems acústicos, que sofre consideravelmente os efeitos dos atrasos de propagação no meio [Brady and Catipovic 1994], afetando o desempenho da rede. Devido ao alto custo para aquisição de equipamentos mais robustos [Benson et al. 2010], modems de baixo custo são mais acessíveis para construir plataformas de testes e formar redes mais densas, sendo necessária uma boa compreensão acerca de seu funcionamento e possíveis impactos que a utilização destes equipamentos podem causar no desempenho e performance da rede. Neste contexto, este trabalho tem por objetivo analisar a performance da comunicação acústica a partir da comparação entre as taxas de transmissão teóricas (utilizadas em experimentos simulados) e taxas de transmissão reais (obtidas em testes de campo) de um modem de baixo custo. Nos experimentos, optouse pela utilização do modem acústico SAM-1 [Star 2016], devido principalmente ao seu custo reduzido comparado a outros equipamentos [Benson et al. 2010] e possibilidade de variação da taxa de transmissão. Nos testes reais realizados, foi possível identificar o comprometimento da taxa de transmissão dos modems, a qual é severamente prejudicada pelos efeitos dos atrasos de propagação no meio.

A principal contribuição deste artigo, além de apresentar informações que auxiliem os pesquisadores com interesse em redes acústicas, mais especificamente nos seus dispositivos de monitoramento, está nos resultados obtidos nos experimentos. Tais resultados demonstram que as taxas máximas de transmissão utilizadas em simulações não refletem as taxas máximas reais quando são consideradas características como a perda de transmissão, o alto nível de ruído, a propagação em múltiplos caminhos, o efeito Doppler e o atraso de propagação variável, existentes em um cenário real. Ademais, os dados 
apresentados possibilitam o desenvolvimento de protocolos MAC (Median Access Control) mais eficientes, permitindo determinar a taxa máxima de transferência de pacotes.

O restante deste artigo está organizado da seguinte forma: a Seção 2 apresenta os trabalhos relacionados, enquanto a Seção 3 apresenta a caracterização do modem SAM1, utilizado nos experimentos. São apresentadas ainda as características de um modem real e os respectivos modelos de taxa de transferência. A Seção 4 apresenta os resultados dos experimentos realizados. Finalmente, na Seção 5, são apresentadas as conclusões e a descrição de trabalhos futuros.

\section{Trabalhos Relacionados}

Alguns trabalhos existentes na literatura indicam que a taxa de transmissão de um modem acústico é de aproximadamente $10 \mathrm{~kb} / \mathrm{s}$. Segundo Yackoski et al. [Yackoski and Shen 2008], a taxa de transmissão de um modem utilizado nos experimentos simulados é definida como sendo de aproximadamente $15 \mathrm{~kb} / \mathrm{s}$, com a velocidade de propagação de $1.500 \mathrm{~m} / \mathrm{s}$ (padrão em comunicações aquáticas que utilizem o canal acústico como meio de transmissão). Vale ressaltar que esta taxa em bits por segundo corresponde ao valor teórico de um modem utilizado em simulações de laboratório.

O mesmo ocorre no trabalho de Ma et al. [Ma and Lou 2011], no qual os autores constroem um algoritmo de agendamento para lidar com as incertezas espaço-temporais geradas pelos longos atrasos de propagação em redes acústicas. No trabalho, os autores também adotam como parâmetro a taxa teórica de transferência do modem de $15 \mathrm{~kb} / \mathrm{s}$, além de outros parâmetros adicionais como velocidade de propagação do sinal acústico, tamanho do pacote de dados e duração do atraso de transmissão dos pacotes. Gan et al. [Guan et al. 2011] projetaram um algoritmo baseado em agendamento que leva vantagem sobre os longos atrasos dos sinais em ambiente acústico, mas também assumem a mesma taxa de transmissão do modem de $15 \mathrm{~kb} / \mathrm{s}$.

Park et al. [Park and Rodoplu 2007] propõem um protocolo MAC eficiente em termos energéticos, utilizando uma taxa de transmissão do modem de cerca de $5.4 \mathrm{~kb} / \mathrm{s}$. Cabe ressaltar que todos os experimentos foram realizados por meio de simulações, não utilizando modems reais.

Na realidade, estas taxas de transmissão, em sua maioria, não correspondem à taxa real de transmissão. Não se pode pré-determinar a taxa de transmissão máxima, o que pode prejudicar, entre outras situações, o funcionamento e o desempenho da rede. Além disso, o desenvolvimento de protocolos são sensíveis a essas circunstâncias e, portanto, a avaliação de modems reais é primordial para o desenvolvimento e funcionamento de aplicações aquáticas.

A evidência de que as taxas reais de transmissão, em sua maioria não correspondem com à taxa teórica foi identificada nos experimentos realizados por Zhu et al. [Zhu et al. 2013]. Após a realização de diversos testes de campo, as características de um modem real foram identificadas. Os autores utilizaram um dispositivo da série ATM, o modem Teledyne Benthos e, nos experimentos práticos realizados pelo autor, a taxa máxima de transmissão alcançou aproximadamente $800 \mathrm{~b} / \mathrm{s}$. Em condições ideais, a taxa de transmissão de modems desta categoria é 2400 b/s [Benson et al. 2010]. De acordo com os resultados de Zhu et al., os longos preâmbulos e as baixas taxas de trans- 
missão são determinantes no desempenho de redes aquáticas, influenciando o tempo total de transmissão e a probabilidade de colisão dos pacotes.

Preâmbulos são sequências incorporadas ao início do frame da camada física, com a finalidade de sincronização, detecção do sinal e estimativa do canal [Zhu et al. 2013]. Quando o atraso gerado pelo tempo de transmissão do preâmbulo é muito curto e a taxa de transmissão é alta, ambos são desconsiderados pela comunidade, como em meios de transmissão via cabo e rede sem fio. No entanto, para sistemas acústicos reais, o tempo de transmissão do preâmbulo é muito longo e não pode ser descartado.

De acordo com os experimentos realizados em [Zhu et al. 2013], o tempo de transmissão encontrado para o preâmbulo em sistemas aquáticos reais foi de $1.5 \mathrm{~s}$, aumentando o tempo de transmissão de pacotes e consequentemente a possibilidade de colisão entre eles. De acordo com os autores, para calcular o tempo de transmissão do pacote é necessário analisar três variáveis:

1. $\mathbf{T}_{p r e}$ : refere-se ao tempo de transmissão do preâmbulo, que é incorporado no tempo de transmissão do pacote;

2. (L): refere-se ao tamanho do pacote em bits;

3. (B): corresponde a taxa de transmissão efetiva do modem em bits por segundo.

A equação (1) [Zhu et al. 2013] apresenta o tempo de transmissão de um pacote, $T_{t x}$, considerando o tempo de transmissão do preâmbulo:

$$
T_{t x}=\frac{L}{B}+T_{p r e}
$$

\section{Caracterização do Modem SAM-1}

O modem acústico SAM-1, utilizado nos experimentos realizados, é caracterizado por uma tecnologia robusta que opera em vários cenários como bacias portuárias, recifes e águas rasas. Este dispositivo trabalha em condições ideais de baixa velocidade no ambiente aquático com um alcance de até $1.000 \mathrm{~m}$. Outras características importantes do modem SAM-1 referem-se à sua sensibilidade ao sinal acústico, que varia de $85 \mathrm{~dB}$ a $125 \mathrm{~dB}$, além da velocidade de comunicação, que varia de 5 a $154 \mathrm{~b} / \mathrm{s}$. Em relação a outros modems acústicos, o SAM-1 destaca-se pelo menor consumo de energia, menor custo e capacidade de utilização em ambientes com ruídos [Star 2016]. O modem SAM-1 é ilustrado na Figura 1.

Existem diversas opções de taxas de transmissão no modem SAM-1. A velocidade de transmissão dos dados acústicos pode ser definida por dois modos distintos de configuração: modem configurado para salto de frequência ou configurado para canal único. Caso o modo de configuração seja salto de frequência, as taxas de transmissão podem ser selecionadas entre 15, 38, 77 e $154 \mathrm{~b} / \mathrm{s}$. Caso o modo escolhido seja canal único, as taxas são de 5, 13 e 38 b/s [Star 2016].

É importante ressaltar que o modo de configuração em canal único (utilizado nos experimentos práticos deste artigo) deve ser selecionado quando a comunicação for realizada entre dois módulos de modems SAM-1. As demais taxas são utilizadas quando o modem SAM-1 estiver em uma comunicação com outro modem capaz de se comunicar em saltos de frequência. 


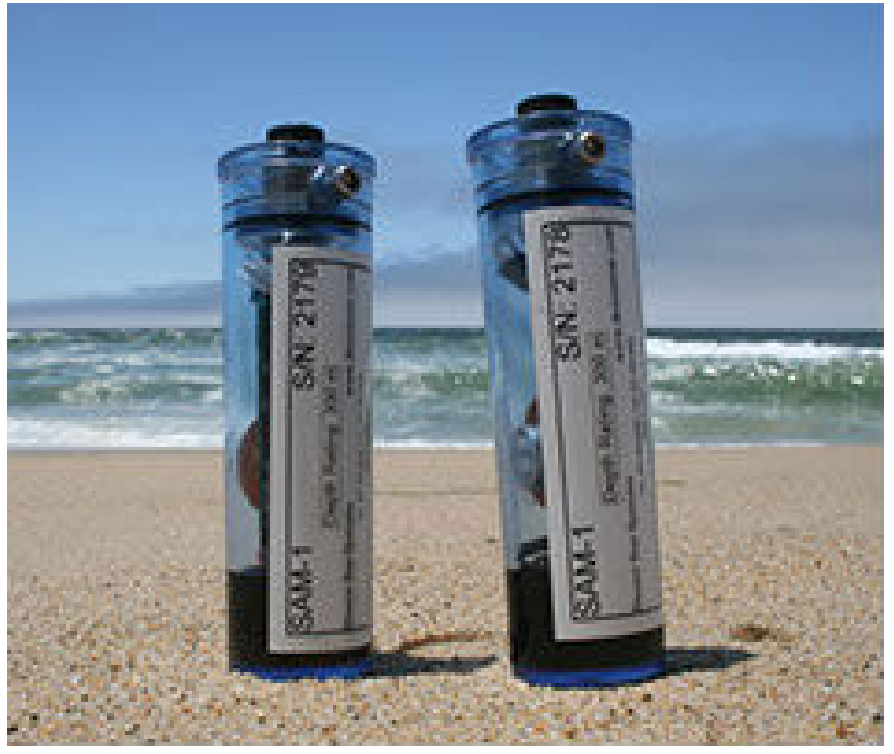

Figura 1. Modens SAM-1 [Star 2016].

A Tabela 1 apresenta as diversas taxas de transmissão configuráveis para o modem SAM-1 [Star 2016]. O fabricante fornece diretivas para a seleção das taxas de transmissão para alguns ambientes típicos. Estas diretivas são apresentadas na Tabela 2.

Tabela 1. Taxas de transmissão do modem SAM-1 [Star 2016].

\begin{tabular}{|l|l|}
\hline Modo de Configuração & Taxa de Transmissão \\
\hline Salto de frequência & S0: $15 \mathrm{~b} / \mathrm{s}$ \\
\hline Salto de frequência & S0: $38 \mathrm{~b} / \mathrm{s}$ \\
\hline Salto de frequência & S0: $77 \mathrm{~b} / \mathrm{s}$ \\
\hline Salto de frequência & S0: $154 \mathrm{~b} / \mathrm{s}$ \\
\hline Canal-único & S0: $5 \mathrm{~b} / \mathrm{s}$ \\
\hline Canal-único & $\mathrm{S} 0: 13 \mathrm{~b} / \mathrm{s}$ \\
\hline Canal-único & S0: $38 \mathrm{~b} / \mathrm{s}$ \\
\hline
\end{tabular}

\section{Resultados Experimentais}

Os experimentos apresentados nesta seção foram realizados em um cenário real, dentro de uma piscina. Foi utilizado um par de modems, modelo SAM-1, distantes $2 \mathrm{~m}$ um do outro, conforme pode ser observado na Figura 2. A rotina de transmissão e recepção foi realizada por ambos os modems, ora agindo como transmissor, ora como receptor.

Utilizou-se do envio de pacotes de dados de diversos tamanhos, variando de 2 a 32 bytes (aumentando sempre de 2 em 2 bytes). No que se refere às taxas de transferência do modem, foram utilizadas as taxas de 5,13 e 38 b/s. O nível de sensibilidade à ruído foi definido em $109 \mathrm{~dB}$. Para cada taxa de transferência do modem, foram realizadas quatro repetições dos experimentos.

As Figuras 3, 4 e 5 apresentam os gráficos que demonstram as médias das porcentagens de bytes dos pacotes entregues para cada uma das três taxas de transferência possíveis (variando o tamanho do pacote).

Nos experimentos realizados, é possível observar que quando as taxas de 5 e $13 \mathrm{~b} / \mathrm{s}$ são selecionadas há alta probabilidade de a entrega do pacote ser feita com sucesso. Re- 
Tabela 2. Diretrizes para seleção de velocidade para alguns ambientes típicos Adaptado de [Star 2016].

\begin{tabular}{|l|l|l|}
\hline \multicolumn{1}{|c|}{ Ambiente } & \multicolumn{1}{|c|}{ Velocidade Máxima } & \multicolumn{1}{c|}{ Descrição } \\
\hline Lagos & $\begin{array}{l}\text { Canal Único: S5-S6 } \\
\text { Salto de Frequência: S2-S3 }\end{array}$ & $\begin{array}{l}\text { Um ’lago típico", ou seja, enlamado e com uma } \\
\text { depressão levemente inclinada no solo, com pouca } \\
\text { superfície de eco. Ecos não tem duração longa, então } \\
\text { altas velocidades podem ser usadas. }\end{array}$ \\
\hline Piscinas e Tanques & $\begin{array}{l}\text { Canal Único: S4 } \\
\text { Salto de Frequência: S0 }\end{array}$ & $\begin{array}{l}\text { Piscinas e tanques exibem ecos muito persistentes. } \\
\text { Somente operações em velocidades mínimas são } \\
\text { possíveis. }\end{array}$ \\
\hline Recifes Superficiais & $\begin{array}{l}\text { Canal Único: S5 } \\
\text { Salto de Frequência: S1-S2 }\end{array}$ & $\begin{array}{l}\text { O som tende a saltar do fundo rochoso e superfície } \\
\text { do mar, criando ecos moderados e exigindo, portanto, } \\
\text { a velocidade moderada. }\end{array}$ \\
\hline Águas Profundas & $\begin{array}{l}\text { Canal Único: S6 } \\
\text { Salto de Frequência: S2-S3 }\end{array}$ & $\begin{array}{l}\text { Há poucos ecos em águas profundas, sendo assim, } \\
\text { altas velocidades são possíveis. }\end{array}$ \\
\hline Portos & $\begin{array}{l}\text { Canal Único: S4-S5 } \\
\text { Salto de Frequência: S0-S2 }\end{array}$ & $\begin{array}{l}\text { As superfícies lisas e resistentes de navios e docas } \\
\text { de persistência moderada à forte. }\end{array}$ \\
\hline
\end{tabular}

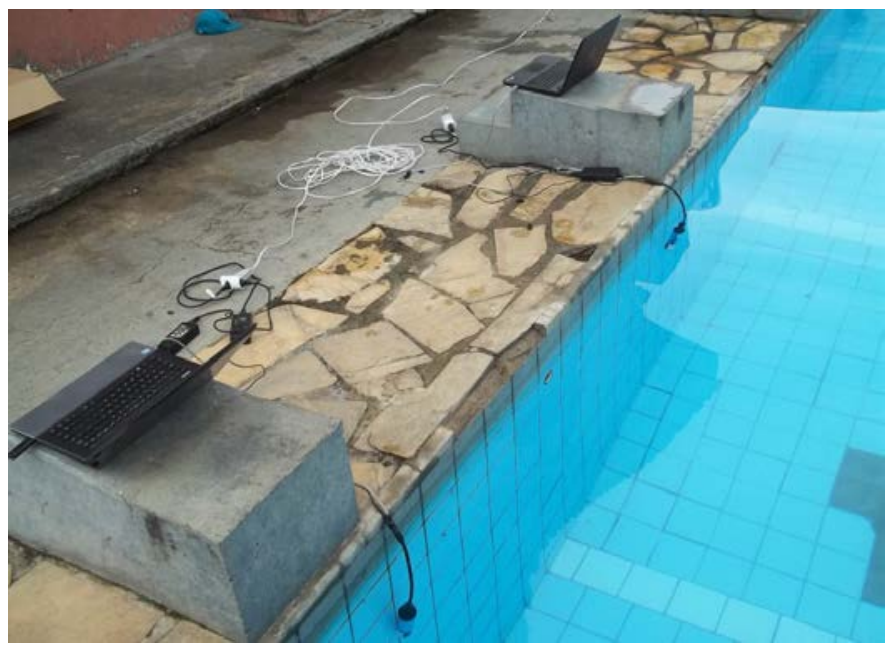

Figura 2. Cenário do Experimento.

sultados mostram uma taxa média de entrega superior a $90 \%$ na maior parte dos cenários com variação do tamanho do pacote. Já considerando a taxa máxima de transmissão do modem (38 b/s), pacotes pequenos (com 2 a 10 bytes) obtiveram taxa média de entrega inferior a $70 \%$. É de consenso comum que pacotes menores tem menos chances de causar colisões no meio. Contudo, conforme análise apresentada por Le et. al. [Le et al. 2013], caso o tempo de início da transmissão destes pacotes não seja cuidadosamente agendado por um protocolo MAC, pode degradar gravemente a performance da rede.

Como apresentado na Seção 2, a presença de preâmbulos longos e baixas taxas de transmissão aumenta consideravelmente o tempo para transmissão de pacotes em redes acústicas aquáticas equipadas com modems reais. Nos experimentos realizados neste trabalho, foi possível observar os efeitos do atraso de propagação do meio acústico no tempo para transmissão de pacotes entre os modems. A disparidade entre as taxas teóricas estimadas e as taxas reais do modem SAM-1 acarretaram em uma substancial diferença no tempo final para entrega dos pacotes. As Figuras 6, 7 e 8 apresentam as médias dos tempos de transmissão real comparadas com os tempos de transmissão ideal de pacotes no modem SAM-1 para cada uma das taxas de transmissão configuráveis do modem (5, 


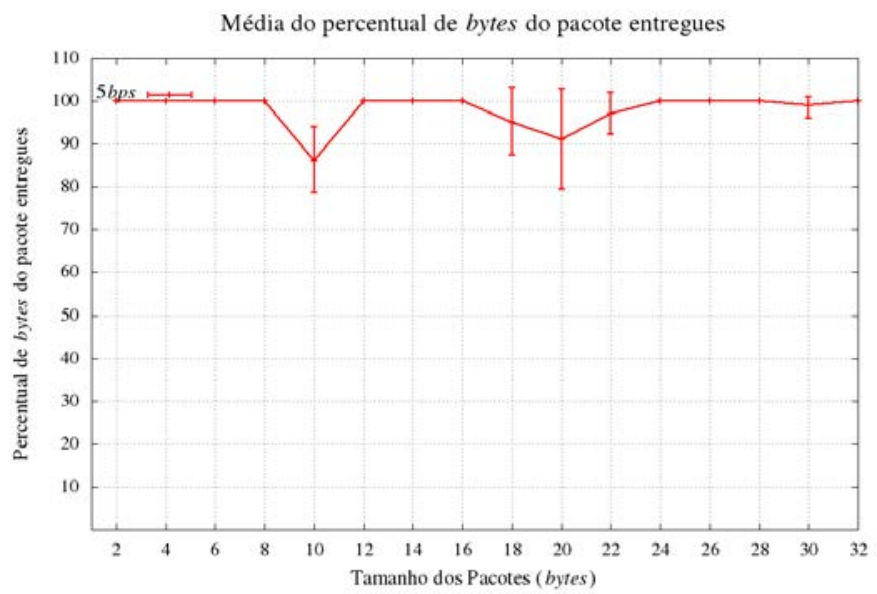

Figura 3. Média do percentual de bytes do pacote entregues ( $5 \mathrm{~b} / \mathrm{s})$.

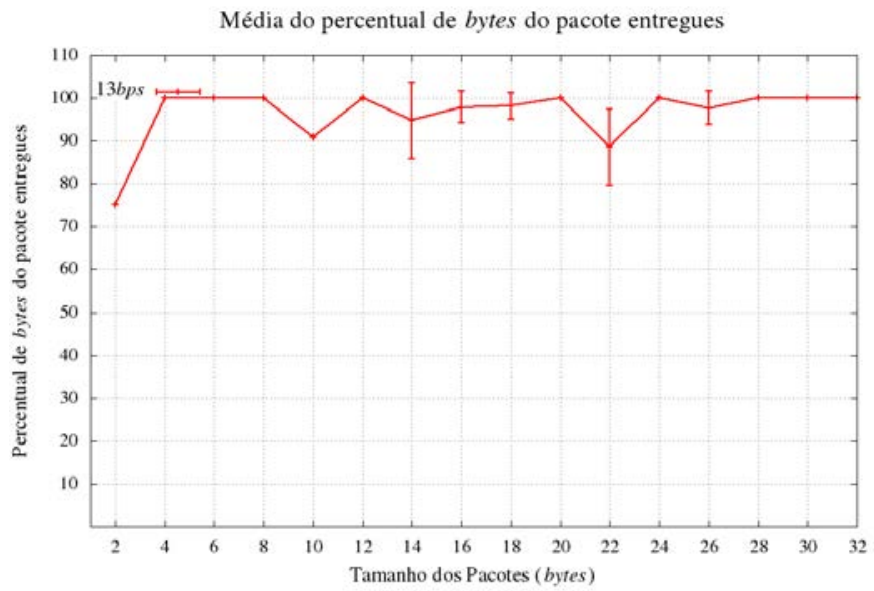

Figura 4. Média do percentual de bytes do pacote entregues (13 b/s).

13 e 38 b/s) e com variação do tamanho do pacote para cada velocidade. A Tabela 3 apresenta o resultado da taxa real obtida em cada transmissão realizada, considerando todos os pacotes transmitidos.

É possível observar uma grande disparidade entre o tempo de transmissão ideal e o tempo de transmissão real de pacotes, principalmente à medida que o tamanho do pacote aumenta. Como exemplo, conforme apresentado no gráfico da Figura 6, um pacote de 32 bytes em condições ideais demoraria aproximadamente $50 \mathrm{~s}$ com uma taxa de transferência de $5 \mathrm{~b} / \mathrm{s}$ para ser entregue. Em condições reais, este tempo aumenta para quase $90 \mathrm{~s}$. A taxa de transmissão real do modem acústico também foi aferida. O modem SAM-1 alcançou no máximo 50\% de sua taxa de transmissão máxima, demonstrando claramente que as taxas máximas de transmissão utilizadas em simulações não refletem as taxas máximas reais, uma vez considerados todos os efeitos de perda de propagação inerentes aos ambientes reais.

\section{Conclusão}

Neste trabalho, foi realizada a avaliação de enlaces de redes acústicas aquáticas utilizando um modem de baixo custo, modelo SAM-1. O principal objetivo dos experimentos é de- 


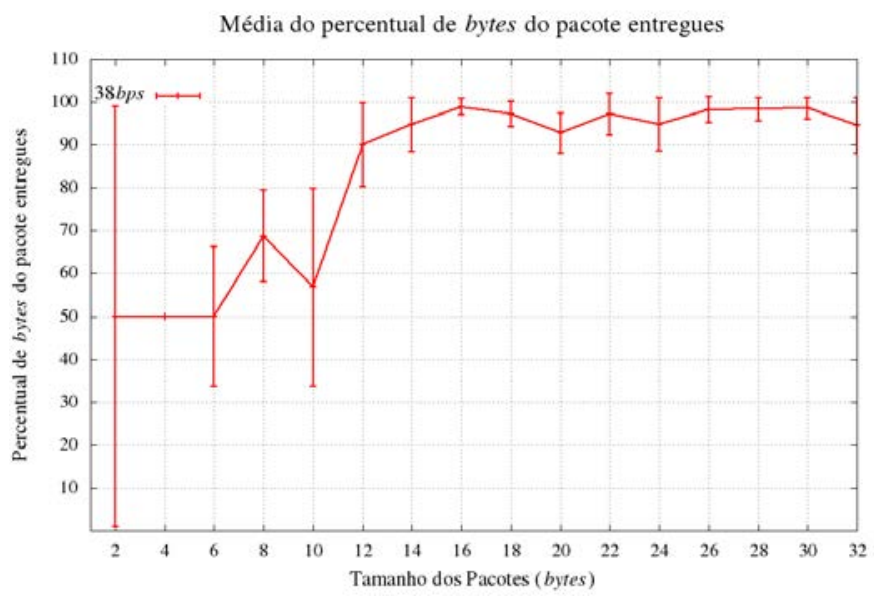

Figura 5. Média do percentual de bytes do pacote entregues ( $38 \mathrm{~b} / \mathrm{s})$.

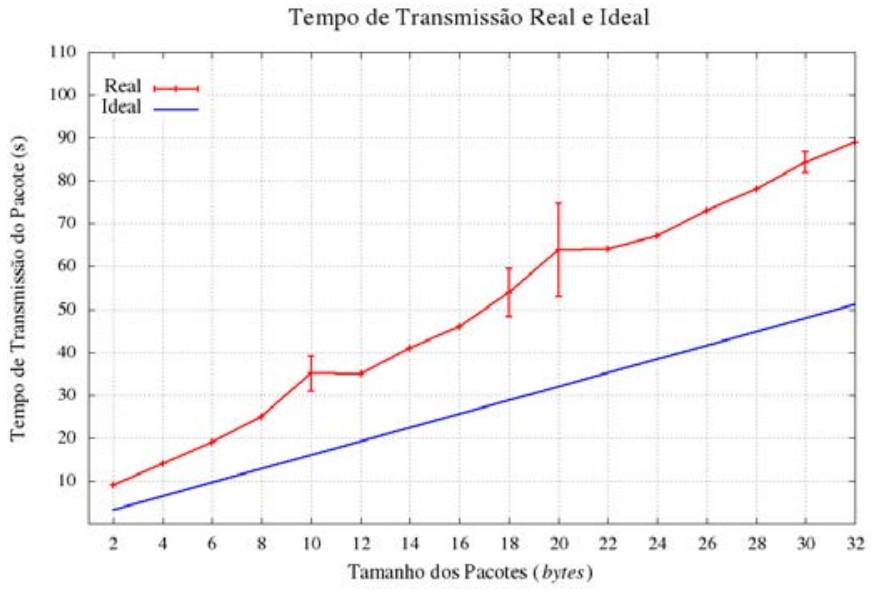

Figura 6. Tempo de Transmissão Real x Tempo de Transmissão Ideal (5 b/s).

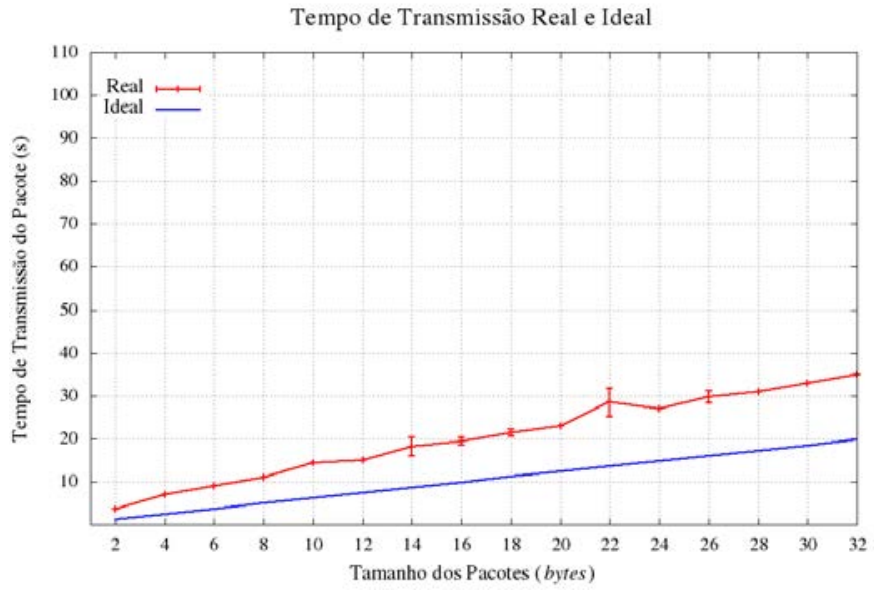

Figura 7. Tempo de Transmissão Real x Tempo de Transmissão Ideal (13 b/s).

monstrar que as taxas máximas de transmissão utilizadas em simulações não refletem as taxas máximas reais quando são consideradas características como a perda de trans- 


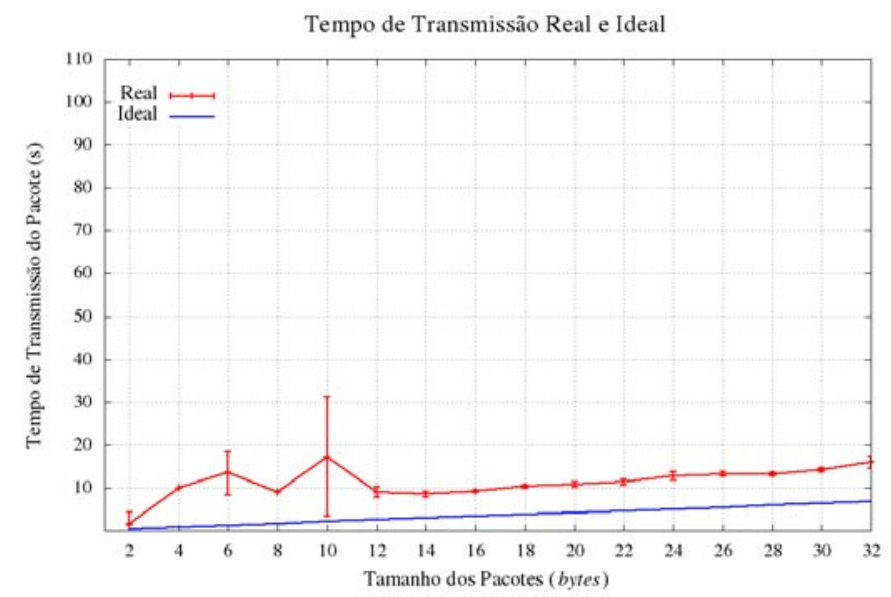

Figura 8. Tempo de Transmissão Real x Tempo de Transmissão Ideal (38 b/s).

Tabela 3. Taxa máxima de transmissão real obtida.

\begin{tabular}{|c|c|c|c|}
\hline Pacote (em bytes) & Taxa Real (5 b/s) & Taxa Real (13 b/s) & Taxa Real (38 b/s) \\
\hline 2 & $1,78 \mathrm{~b} / \mathrm{s}$ & $4,57 \mathrm{~b} / \mathrm{s}$ & $10,67 \mathrm{~b} / \mathrm{s}$ \\
\hline 4 & $2,29 \mathrm{~b} / \mathrm{s}$ & $4,57 \mathrm{~b} / \mathrm{s}$ & $3,20 \mathrm{~b} / \mathrm{s}$ \\
\hline 6 & $2,53 \mathrm{~b} / \mathrm{s}$ & $5,33 \mathrm{~b} / \mathrm{s}$ & $3,56 \mathrm{~b} / \mathrm{s}$ \\
\hline 8 & $2,56 \mathrm{~b} / \mathrm{s}$ & $5,82 \mathrm{~b} / \mathrm{s}$ & $7,11 \mathrm{~b} / \mathrm{s}$ \\
\hline 10 & $2,29 \mathrm{~b} / \mathrm{s}$ & $5,59 \mathrm{~b} / \mathrm{s}$ & $4,63 \mathrm{~b} / \mathrm{s}$ \\
\hline 12 & $2,74 \mathrm{~b} / \mathrm{s}$ & $6,40 \mathrm{~b} / \mathrm{s}$ & $10,65 \mathrm{~b} / \mathrm{s}$ \\
\hline 14 & $2,73 \mathrm{~b} / \mathrm{s}$ & $6,18 \mathrm{~b} / \mathrm{s}$ & $13,19 \mathrm{~b} / \mathrm{s}$ \\
\hline 16 & $2,78 \mathrm{~b} / \mathrm{s}$ & $6,58 \mathrm{~b} / \mathrm{s}$ & $14,07 \mathrm{~b} / \mathrm{s}$ \\
\hline 18 & $2,67 \mathrm{~b} / \mathrm{s}$ & $6,72 \mathrm{~b} / \mathrm{s}$ & $13,98 \mathrm{~b} / \mathrm{s}$ \\
\hline 20 & $2,51 \mathrm{~b} / \mathrm{s}$ & $6,96 \mathrm{~b} / \mathrm{s}$ & $14,80 \mathrm{~b} / \mathrm{s}$ \\
\hline 22 & $2,75 \mathrm{~b} / \mathrm{s}$ & $6,17 \mathrm{~b} / \mathrm{s}$ & $15,51 \mathrm{~b} / \mathrm{s}$ \\
\hline 24 & $2,87 \mathrm{~b} / \mathrm{s}$ & $7,11 \mathrm{~b} / \mathrm{s}$ & $15,11 \mathrm{~b} / \mathrm{s}$ \\
\hline 26 & $2,85 \mathrm{~b} / \mathrm{s}$ & $6,99 \mathrm{~b} / \mathrm{s}$ & $15,71 \mathrm{~b} / \mathrm{s}$ \\
\hline 28 & $2,87 \mathrm{~b} / \mathrm{s}$ & $7,23 \mathrm{~b} / \mathrm{s}$ & $16,94 \mathrm{~b} / \mathrm{s}$ \\
\hline 30 & $2,85 \mathrm{~b} / \mathrm{s}$ & $7,27 \mathrm{~b} / \mathrm{s}$ & $16,88 \mathrm{~b} / \mathrm{s}$ \\
\hline 32 & $2,88 \mathrm{~b} / \mathrm{s}$ & $7,31 \mathrm{~b} / \mathrm{s}$ & $16,05 \mathrm{~b} / \mathrm{s}$ \\
\hline
\end{tabular}

missão, o alto nível de ruído, a propagação em múltiplos caminhos, o efeito Doppler e o atraso de propagação variável, existentes em um cenário real. Conforme demonstrado nos experimentos práticos realizados neste trabalho, a taxa de transmissão real do modem acústico SAM-1 foi, no melhor dos cenários, $50 \%$ da taxa de transmissão teórica, independente da velocidade configurada no equipamento. Como consequência levou-se, em muitos casos, o dobro do tempo que se levaria em condições normais para transmitir um pacote, independente de seu tamanho.

Acredita-se que os dados apresentados neste artigo servirão de auxílio na compreensão e na modelagem do comportamento dos modems acústicos, o que permitirá o projeto de protocolos sensíveis à necessidade de pré-determinar a taxa de transferência máxima e probabilidade de colisão na rede. Espera-se ainda que os resultados possam auxiliar o desenvolvimento de aplicações em diversas áreas, como monitoramento de lagos, exploração de petróleo e monitoramento da infra-estrutura na indústria petrolífera.

Para trabalhos futuros, planeja-se o desenvolvimento de protocolos que se baseiem nas taxas de transmissão reais, permitindo o funcionamento correto da rede. 


\section{Agradecimentos}

À CAPES, CNPq e FAPEMIG, pelas bolsas e apoio financeiro.

\section{Referências}

Benson, B., Li, Y., Kastner, R., Faunce, B., Domond, K., Kimball, D., and Schurgers, C. (2010). Design of a Low-cost, Underwater Acoustic Modem for Short-range Sensor Networks. In OCEANS 2010 IEEE - Sydney, pages 1-9.

Brady, D. P. and Catipovic, J. A. (1994). Acoustic Local Area Networks. US Patent $5,303,207$.

Catipovic, J., Brady, D., and Etchemendy, S. (1993). Development of Underwater Acoustic Modems and Networks. Oceanography, 6(3):112-119.

Guan, Y., Shen, C.-C., and Yackoski, J. (2011). MAC Scheduling for High Throughput Underwater Acoustic Networks. In Wireless Communications and Networking Conference (WCNC), 2011 IEEE, pages 197-202. IEEE.

Le, S. N., Zhu, Y., Peng, Z., Cui, J.-H., and Jiang, Z. (2013). PMAC: a Real-world Case Study of Underwater MAC. In Proceedings of the Eighth ACM International Conference on Underwater Networks and Systems, page 2. ACM.

Ma, J. and Lou, W. (2011). Interference-aware Spatio-temporal Link Scheduling for Long Delay Underwater Sensor Networks. In Sensor, Mesh and Ad Hoc Communications and Networks (SECON), 2011 8th Annual IEEE Communications Society Conference on, pages 431-439. IEEE.

Park, M. K. and Rodoplu, V. (2007). UWAN-MAC: An Energy-efficient MAC Protocol for Underwater Acoustic Wireless Sensor Networks. Oceanic Engineering, IEEE Journal of, 32(3):710-720.

Star, D. (2016). Manual do Fabricante. Acessado em fevereiro de 2016. Disponível em: http://desertstar.com/product/sam-1/.

Stojanovic, M. and Preisig, J. (2009). Underwater Acoustic Communication Channels: Propagation Models and Statistical Characterization. Communications Magazine, IEEE, 47(1):84-89.

Vieira, L. F., Vieira, M. A., Pinto, D., Nacif, J. A. M., Viana, S. S., and Vieira, A. B. (2012). HydroNode: An Underwater Sensor Node Prototype for Monitoring Hydroelectric Reservoirs. In Proceedings of the Seventh ACM International Conference on Underwater Networks and Systems, page 43. ACM.

Yackoski, J. and Shen, C.-C. (2008). UW-FLASHR: Achieving High Channel Utilization in a Time-based Acoustic MAC Protocol. In Proceedings of the Third ACM International Workshop on Underwater Networks, pages 59-66. ACM.

Zhu, Y., Jiang, Z., Peng, Z., Zuba, M., Cui, J.-H., and Chen, H. (2013). Toward Practical MAC Design for Underwater Acoustic Networks. In INFOCOM, 2013 Proceedings IEEE, pages 683-691. IEEE. 